\title{
Prediction of the electrical load of the power system using neural networks
}

\author{
$D$ I Nabiullin* and $R N$ Balobanov
}

Krasnoselskaya st., 51, Kazan, Rep. Tatarstan, 420034, Russia

\begin{abstract}
Prediction of the electrical load schedule of an electrical system is an important aspect for determining electrical loads, which ensures the correct selection and cost-effective operation of reactive power compensation devices and voltage control devices, as well as relay protection and automation. This article discusses methods for predicting electrical load using an artificial neural network. The problems of choosing the optimal architecture and algorithm of neural network training are considered. The methods of the best forecast accuracy are determined. A genetic algorithm based on the group method of data handling was chosen as the main calculation.
\end{abstract}

\section{Introduction}

Prediction of power consumption and the corresponding mode parameters is an important task in planning and controlling the operating modes, ensuring the correct operation of the system. This process can be implemented in several ways: a short-term forecast (from a few seconds to an hour), an operational forecast (from an hour to a week), a medium-term forecast (from a week to a year), a long-term forecast (from a year to 20 years). They can be implemented using various methods, such as: analytical, working on the basis of the identified patterns and allowing to build a mathematical model expressed by a system of equations, or statistical, based on probability theory, the use of which is appropriate for stable energy objects. Also distinguished expert systems: intelligent logical control systems and intelligent controllers [1].

Artificial neural networks are devices based on parallel processing of information by all links. These links can be trained in the process of work and summarize the accumulated knowledge. Neural networks inherent features of artificial intelligence. The most popular artificial neural network architecture for predicting electrical load is the forward propagation architecture. This network uses continuously evaluated features and teacher training. The actual numerical data of the weights assigned to the inputs of the elements is determined by comparing the historical data (for example, time and weather) with the given outputs (for example, the predicted load data) during training. Artificial neural networks with uncontrolled training do not require prior operational training. In view of the fact that at present the priority direction in the field of modernization of the electric power industry is the development of the Smart Grid, so the artificial intelligence methods will be used in the study to solve the problem of predicting electrical loads.

The balance of production and consumption of electricity is the basis of the technological stability of the energy system, it determines the system reliability and uninterrupted operation of it. In case of violations of the balance condition, the quality of electric power deteriorates (change in the parameters of the frequency and voltage of the network), which reduces the efficiency of the power-consuming equipment of the final consumers. There are quite a few economic reasons for the need for high-quality forecasting. Accurate calculations ensure optimal load distribution between the power stations of the power grid and improve the quality of electricity. Forecasting and planning indicators of electricity consumption for large consumers allows you to manage the cost of purchasing electricity through regulating the loading of equipment through managing production processes, translating the main volumes of electricity consumption in hours with the lowest cost, thereby reducing the cost of production and the amount of payments to power supply organizations. [2]

\section{Algorithms for prediction}

To date, many algorithms have been developed for analyzing and forecasting time series. At the same time, none of them can be called universal. Most of the algorithms do not meet the requirement of high prediction accuracy in a constantly changing state of the electricity market. Due to the increased power of personal computers, it became possible to use neural network algorithms for analyzing time series. Operations on the matrix data describing the passage of a signal through a neural network on a modern computer are performed in a matter of seconds. Artificial neural

Corresponding author: dinar91121@mail.ru 
networks are a powerful tool for pattern recognition, approximation and forecasting of time series.

The construction of a neural network is solved in two stages: the choice of the type (architecture) of the neural network and the selection of weights (training) of the neural network.

At the first stage are selected:

the types of neurons that must be used (number of inputs, transfer functions);

methods of connecting neurons with each other;

input and output signals of the neural network.

There are several dozens of different neural network architectures, and the effectiveness of many of them is mathematically proven. The most popular and studied architectures are the multilayer perceptron, the neural network with general regression, Kohonen neural networks, etc.

To solve the problem with the help of artificial neural networks, it is necessary to select the neural network configuration and form the environment in which it will work

This neural network. Under the formation of the environment refers to the implementation of the following steps:

- creation of a training problem book;

- training of the neural network;

- testing an artificial neural network.

\section{The choice of the main method}

Currently, the so-called group method of data handling (GMDH) method, which is a further development of the regression analysis method, is becoming increasingly popular for forecasting problems. It is based on some principles of the theory of learning and self-organization, in particular on the principle of "selection", or directional selection. The method implements the problem of synthesizing optimal models of high complexity, adequate complexity of the object under study (here, models are understood as a system of regression equations). So, GMDH algorithms, built according to the mass selection scheme, carry out a search of possible functional descriptions of the object. Various combinations of input and intermediate variables are considered, and a model is constructed for each combination, and the most regular variables are used when building the selection series. The concept of regularity is one of the main ones in the GMDH method [7-12].

Below is the perceptron, which is presented as the main neural network architecture. This neural network works on polynomial combinatorics.

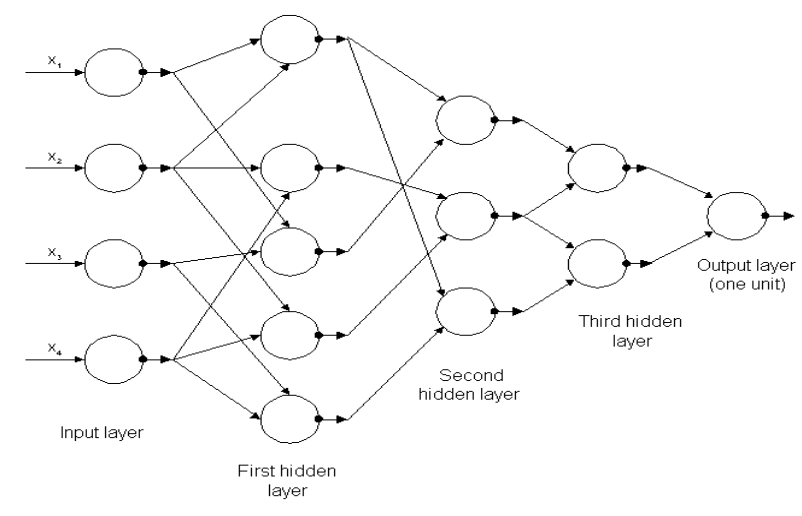

Fig. 1. Neural network architecture of group method data handling.

1. The regularity is determined by the minimum of the mean square error of the variables on a separate test data sequence (the initial series is divided into training and test sequences). In some cases, the correlation coefficient is used as an indicator of regularity. Rows are built as long as the regularity rises, that is, the error decreases or the correlation coefficient increases. Thus, from the entire set of models is chosen such that is optimal in terms of the selected criteria.

In the course of the work, the program GMDH Shell was used to create artificial neural networks, which made it possible to implement a long-term electrical load schedule.

In the course of work in the GMDH Shell program, a load schedule was created by months with further forecasting until 2020. The method is based on the recursive selection of models on the basis of which more complex models are built. The accuracy of the simulation at each next recursion step is increased due to the complexity of the model.

Given the observations for a certain period.

1. A general view of the iterated models is selected, the so-called support functions. Often used by the Kolmogorov-Gabor polynomial:

$$
\begin{aligned}
& Y\left(x_{1}, \ldots, x_{n}\right)=a_{0}+\sum_{i=1}^{n} a_{i} x_{i}+ \\
& +\sum_{i=1}^{n}\left(\sum_{j=i}^{n} a_{i j} x_{i} x_{j}\right)+\sum_{i=1}^{n}\left[\left(\sum_{j=i}^{n}\right)\left(\sum_{k=j}^{n} a_{i j k} x_{i} x_{j} x_{k}\right)\right]+\ldots
\end{aligned}
$$

The choice of polynomials is due to the property that according to the Weierstrass theorem, any continuous function on a finite interval can be represented with an arbitrarily high accuracy as a polynomial of a certain degree. The number of coefficients in this case determines the complexity of the model.

2. Using support functions, various models are constructed for some or all of the arguments. For example, polynomials with one variable, polynomials with all possible pairs of variables, polynomials with all possible triples of variables, and so on, a polynomial with all variables are constructed. For each model, its coefficients are determined a_(ij...k) method of regression analysis. 


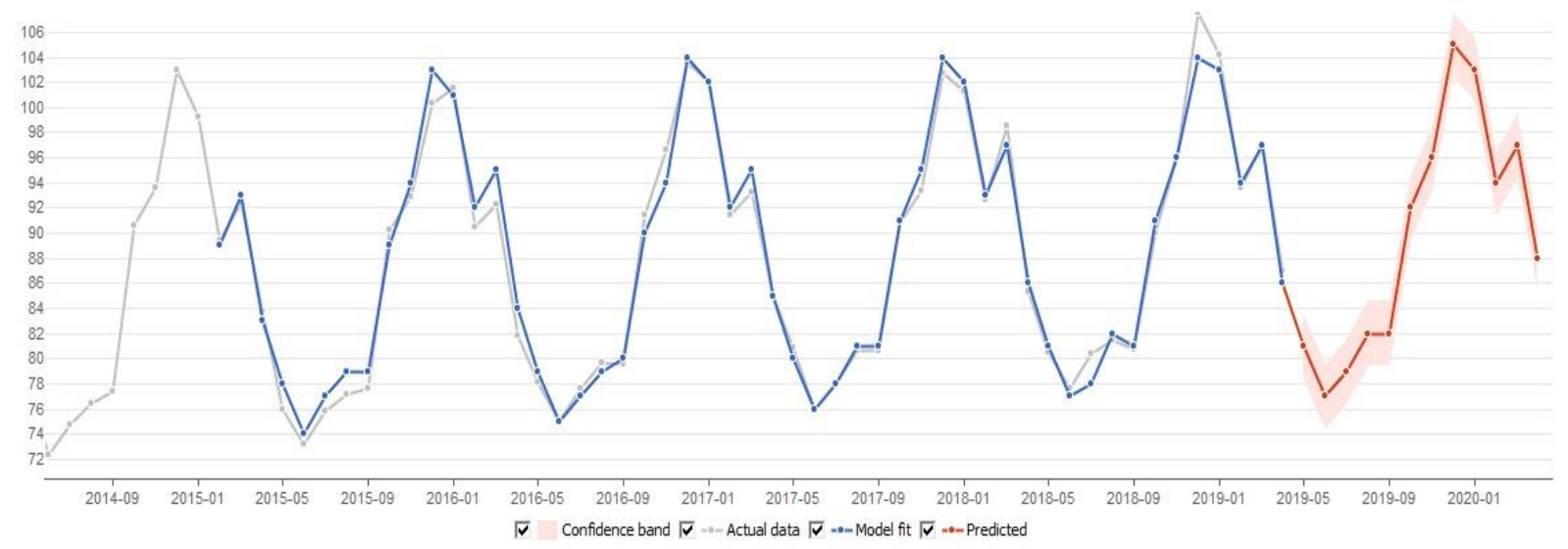

Fig. 2. The data of the load curve of the electrical system from 09.2014-01-2020 year.

3. Among all models, several (from 2 to 10) are selected. The quality of the models is determined by the coefficient of determination, or the standard deviation of the error, or by the correlation of $\mathrm{Y}$ and the original data.

4. If a "suitable" model is found or the maximum permissible complexity of the models is reached, the algorithm ends.

5. Otherwise, the models found in the 3rd step are used as arguments $\left(\mathrm{x} \_1, \ldots \mathrm{x} \_\mathrm{n}\right)$ for the reference functions of the next iteration stage (go to the 2 nd point). That is, already found models are involved in the formation of more complex ones [6-9].

As a training sample (data), we used the archive database on the months of the electrical load of the power system of Russia, the average temperature for 2009-2019 [11].

The figure shows a graph of actual and predicted values for each month of 2014, where you can clearly see the accuracy of the forecast up to 2020. (Fig. 2).

Table 1. Actual and predicted data.

\begin{tabular}{|c|c|c|}
\hline Data & Actual data $\left(\right.$ bil $\left.\mathrm{kW}^{*} \mathrm{~h}\right)$ & Predict \\
\hline 01.2019 & 104.2 & 103 \\
\hline 02.2019 & 93.6 & 94 \\
\hline 03.2019 & 97.1 & 97 \\
\hline 04.2019 & 87 & 86 \\
\hline 05.2019 & 81.9 & 81 \\
\hline 06.2019 & - & 77 \\
\hline 07.2019 & - & 79 \\
\hline 08.2019 & - & 82 \\
\hline 09.2019 & - & 82 \\
\hline 10.2019 & - & 92 \\
\hline 11.2019 & - & 96 \\
\hline 12.2019 & - & 105 \\
\hline 01.2020 & - & 103 \\
\hline
\end{tabular}

The results of the conducted studies show that the accuracy of the forecast increases every month; this is due to the inclusion of the predicted value of the electrical load in the retrospective series for further iteration of the calculation.

\section{Conclusion}

Comparison of the results of the predicted values of electrical load with its real values showed that the calculated forecast error does not exceed the allowable value and the accuracy of the forecast using the neural network model is much higher. Therefore, an increase in the accuracy of the forecast even by the tenths of a percent can lead to a significant reduction in the cost of paying for deviations from the plan for the supply of electricity. It turns out that the task of forecasting is of high relevance for a large number of entities operating in the wholesale electricity market: for guaranteeing suppliers serving consumers of entire regions, independent energy sales organizations serving individual industrial enterprises in various regions of Russia, and for large business entities buying electricity in the wholesale market for their own consumption. Therefore, high-quality forecasting of electricity consumption for the subjects of the Russian wholesale market is economically feasible and in current highly competitive conditions gets increasing relevance

\section{References}

1. Neural networks (2018) URL http://statsoft.ru/home/textbook/modules/stneunet.

2. G.P. Shumilova, N.E. Gotman, T.B. Startseva, Prediction of electrical loads in the operational management of electrical energy systems based on neural network structures, 88 (2008)

3. B.V. Papkov, Reliability and efficiency of power supply: tutorial, 210 (1996)

4. C.E. Kuznecov, Forecasting power consumption using neural networks (2016)

5. K.L. Solomakho, Use of the principal components method to predict the electricity consumption of the power supply company, 141 (2015)

6. Group method if data handling (2019) https://en.wikipedia.org/wiki/GMDH 
7. A.M. Abdurakhmanov, M.V. Volodin, Ye.Yu. Zybin, Methods for predicting power consumption in distribution networks, 18-20 (2016)

8. V.Z. Manusov, S.V. Khokhlova., Comparative analysis of two models of electrical load forecasting of industrial enterprises based on regression analysis and artificial neural networks, Scientific messenger NGTU, 1, 11-13 (2008)

9. Wiener series (2019) URL https://en.wikipedia.org/wiki/Wiener_series

10. N.A. Serebryakov, S.O. Khomutov, Improving the quality of short-term electrical load forecasting for a group of points of supply for agricultural producers Using a multi-layer perceptron, 90 (2016)

11. System Operator of the Unified Energy System (2019) URL https://so-ups.ru/

12. I.Yu. Alekseyeva, Improving the reliability of electric power systems based on neural technologies, 15 (2016)

13. Group method of data handling (2019) URL http://www.gmdh.net/ 